\title{
Formation and Large-Scale Patterns of Filament Channels and Filaments
}

Duncan H Mackay

\begin{abstract}
The properties and large-scale patterns of filament channels and filaments are considered. Initially, the global formation locations of filament channels and filaments are discussed, along with their hemispheric pattern. Next, observations of the formation of filament channels and filaments are described where two opposing views are considered. Finally, the wide range of models that have been constructed to consider the formation of filament channels and filaments over long time-scales are described, along with the origin of the hemispheric pattern of filaments.
\end{abstract}

\section{Global Patterns of Filaments and Filament Channels}

\subsection{Global Patterns}

Solar filaments (a.k.a. prominences) form over a wide range of latitudes on the Sun. These latitudes range from the active latitudes all the way to the polar crown. A typical distribution of solar filaments across the Sun during a period of high magnetic activity can be seen in Figure 1(a). The filaments can be seen in $\mathrm{H} \alpha$ absorption as dark features that bend and weave their way across the solar surface. The long term behavior of solar filaments over many solar cycles may be deduced from archives such as Cartes Synoptiques ${ }^{1}$ (1919-1989 covering 7 solar cycles) or from NOAA Solar Geophysical Data ${ }^{2}$ (1955-2009 covering 6 solar cycles). These data sets show that the number of solar filaments present on the Sun at any one time varies in a manner similar to that of the sunspot number. Over a single Carrington Rotation the

Duncan H Mackay

School of Mathematics and Statistics, University of St Andrews, North Haugh, St Andrews, Fife, KY16 8HB e-mail: dhm@st-and.ac.uk

ftp.ngdc.noaa.gov/STP/space-weather/solar-data/solar-features/prominences-

filaments/filaments/

${ }^{2}$ http://www.ngdc.noaa.gov/stp/solar/sgd.html 
total number of filaments on the Sun varies from 20 at cycle minimum to over 100 at cycle maximum (Zou et al., 2014). There is also a clear latitudinal dependence to the number of filaments. At low latitudes $\left(\lambda<50^{\circ}\right)$ there are more filaments and a stronger variation (10-80 from cycle min to max), while at high latitudes $\left(\lambda>50^{\circ}\right)$ the variation is less strong $(0-10)$.

As magnetic flux is transported across the surface of the Sun, solar filaments are found to migrate over a range of latitudes (McIntosh, 1972; Minarovjech et al., 1998). This migration can be seen in Figure 1(b) where the solar filament butterfly diagram is shown from 1919-1989 (Coffey \& Hanchett, 1998). In contrast to the sunspot butterfly diagram, the filament butterfly diagram shows a more complex structure with two branches of migration. The first branch follows a similar path to that of the sunspot butterfly diagram and extends towards lower latitudes. While it follows a similar path, solar filaments occur over a wider latitude range than sunspots $(\mathrm{Li}, 2010)$. The second branch follows the poleward transport of magnetic flux during the rising phase of the solar cycle and is named the "rush to the poles of polar crown filaments" (Topka et al., 1982; Mouradian and Soru-Escaut, 1994; Shimojo et al., 2006; Pintér et al., 2014). The disappearance of this branch signifies the reversal of the Sun's polar field around solar maximum. Due to this, filaments may be used as a tracer to infer the large-scale pattern of magnetic flux on the Sun.

Based upon the magnetic environment in which they form, filaments may be split into three broad categories (Engvold (1998), see also Chapter 2):

1. Quiescent Filaments: Filaments found in quiet regions of the Sun and around the polar crown (e.g. QF in Figure 1(a)).

2. Intermediate Filaments: Filaments that form around the borders of active regions (IF in Figure 1(a)).

3. Active Region Filaments: Filaments found within the centers of activity nests of multiple pairs of sunspots (ARF in Figure 1(a)).

Observations tend to show that IFs and QFs are larger, much more stable structures with longer lifetimes (weeks to months) compared to ARFs, which are generally unstable with a lifetime of only a few hours to days. In later sections when discussing the possible mechanisms of filament formation, this classification scheme will prove useful in illustrating that different mechanisms may form different types of filaments.

\subsection{Properties of Filament Channels}

Although filaments may form at many locations on the Sun, they always form above Polarity Inversion Lines (PILs, Babcock and Babcock (1955)), which divide regions of positive and negative flux in the photosphere. As discussed in Martin (1998) the existence of a PIL is a necessary, but not sufficient condition for a filament to form. For a filament to form, a filament channel must also exist at the height of 


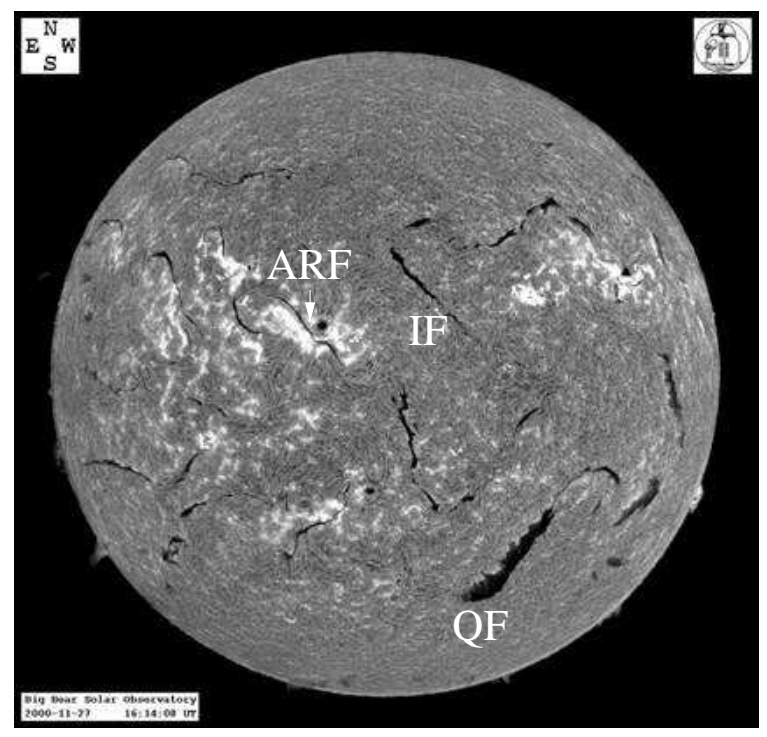

(a)

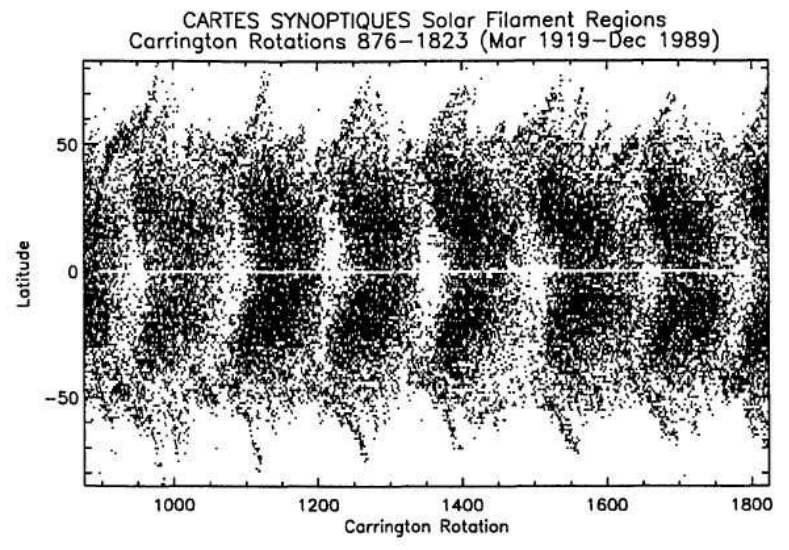

(b)

Fig. 1 (a) Full disk $\mathrm{H} \alpha$ filtergram from Big Bear Solar Observatory on $22^{\text {nd }}$ November 2000. Examples of Quiescent (QF), Intermediate (IF) and Active Region (ARF) filaments are shown. (b) The filament butterfly diagram produced from Cartes Synoptiques data (from Coffey \& Hanchett (1998), Figure 2.)

the chromosphere (Gaizauskas, 1998). Observations show that not every filament channel contains a filament. Due to this, filament channels are more fundamental than the filaments that form within them, where a single channel may survive a succession of filament formations and eruptions. 
The basic magnetic structure of a filament channel is illustrated in Figure 2. The properties of filament channels were first noted by Martres et al. (1966) who described them as a region in the chromosphere around a PIL where the chromospheric fibrils are aligned, (1) anti-parallel to one-another on either side and (2) nearly parallel to the path of the PIL. Foukal (1971a,b) interpreted these fibrils as giving the direction of the local magnetic field in the chromosphere. Due to the anti-parallel alignment of the fibrils, Foukal deduced that the horizontal component of magnetic field must point in the same direction on either side of the channel (Martin et al., 1992, 1994). For a more in depth discussion of the observational properties of filament channels see Chapter 2, Section 3.1. From this fibril alignment it can be deduced that filament channels, the birth ground of filaments, are locations of strong magnetic shear and highly non-potential magnetic fields. Solar filaments which lie in the corona are believed to be embedded in the filament channel field which extends up into the corona. Through Hanle and Zeeman effect measurements (Hyder, 1965; Rust, 1967; Leroy et al., 1983; Leroy, 1989) the existence of a dominant horizontal field has been verified at coronal heights.

Presently it is unclear why channels and their non-potential fields build up along PILs. Karachik \& Pevtsov (2014) showed that PILs with and without filaments have a similar gradient of the magnetic field across the PIL. Understanding filament channel formation is key to our understanding of the evolution of magnetic fields on the Sun and their relationship to eruptive phenomena. By observing and interpreting their formation and evolution, we may examine directly the buildup of magnetic stress and energy required for Space Weather events such as Coronal Mass Ejections. The importance of filament channels to Space Weather can be seen by the paper of Pevtsov et al. (2012) who showed that filament channels without filaments may lead to CMEs. In Section 5 a wide range of models and mechanisms for the development of the strong shear and axial fields in filament channels and filaments will be discussed. In the next section we consider the global properties and formation locations as deduced from $\mathrm{H} \alpha$ observations.

\section{Global Formation Locations}

While filaments and filament channels form at many locations on the Sun, very few studies have considered the exact nature or history of the PILs above which they form. Those studies that have considered this, are mainly restricted to studying large-scale, stable filaments and neglect smaller unstable filaments forming in the centers of activity complexes. Understanding the type of magnetic environment in which filaments and filament channels form is key to understanding the magnetic interactions that lead to the formation of strong magnetic shear.

In the past many classification schemes for filaments and prominences have been developed (d'Azambuja and d'Azambuja (1948) see Chapter 2). One classification scheme (Engvold, 1998) provides a useful distinction between filaments forming inside and outside active regions. However, to understand the role that magnetic fields 


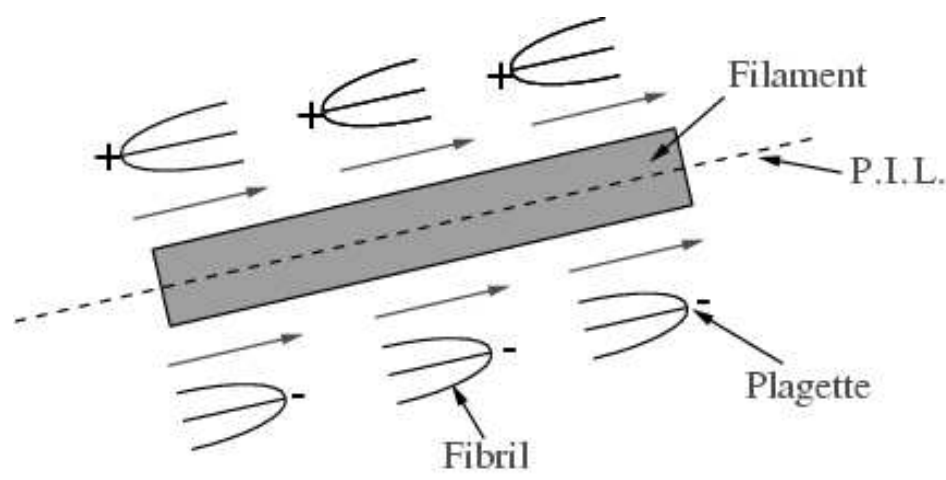

Fig. 2 Schematic of a filament channel with fibrils which lie (1) anti-parallel to one-another on either side of the PIL and (2) nearly parallel to the path of the PIL. The anti-parallel alignment indicates that the magnetic field (arrows) is dominantly horizontal and points in the same direction on either side of the channel.

play in the formation, structure and evolution of filaments it is important to understand the exact type of PIL above which filaments form. One early classification scheme that aims to quantify this (Tang, 1987) splits filaments into two categories. The first category is one in which the filament forms above a PIL lying within a single bipolar unit of flux, and is classified as an "Internal Bipolar Region Filament" (see Figure3a). In the second, the filament forms above a PIL which lies between two separate magnetic bipoles and is called an "External Bipolar Region Filament" (Figure 3b). Observations by Tang (1987) showed that when filaments are classified into these two types, over $60 \%$ of filaments form external to bipolar regions.

In a more recent study Mackay et al. (2008) reconsidered where large, stable solar filaments form. A key aspect of this study was that the authors followed the history and evolution of the PILs underneath filaments before each of the filaments was categorised. To distinguish the different bipole interactions that could lead to the formation of filaments, Mackay et al. (2008) introduced two additional categories in addition to those defined by Tang (1987): "Internal/External Bipolar Regions Filaments" (I/EBR) and "Diffuse Bipolar Region Filaments" (DBR). The I/EBR filaments are defined as filaments that lie above both the internal PIL of a bipole and the external PIL surrounding the bipole (Figure 3c), and therefore could not be classified into the scheme proposed by Tang (1987). In contrast, the DBR filaments are located in essentially a bipolar distribution of flux, but where the polarities defining the bipole did not emerge together. The formation of the bipolar distribution was the result of many flux emergences, coalescences and cancellations such that the polarities on either side of the filament could not be attributed to a single bipole emergence (Figure 3d). Mackay et al. (2008) then consider the bipole interactions leading to the formation of Intermediate and Quiescent filaments over four distinct phases of the solar cycle (two before and two after cycle maximum) .

Of the 603 filaments studied by Mackay et al. (2008), 92\% formed at locations requiring multiple bipole interactions (the breakdown comprised of $62 \%$ EBR, $17 \%$ 


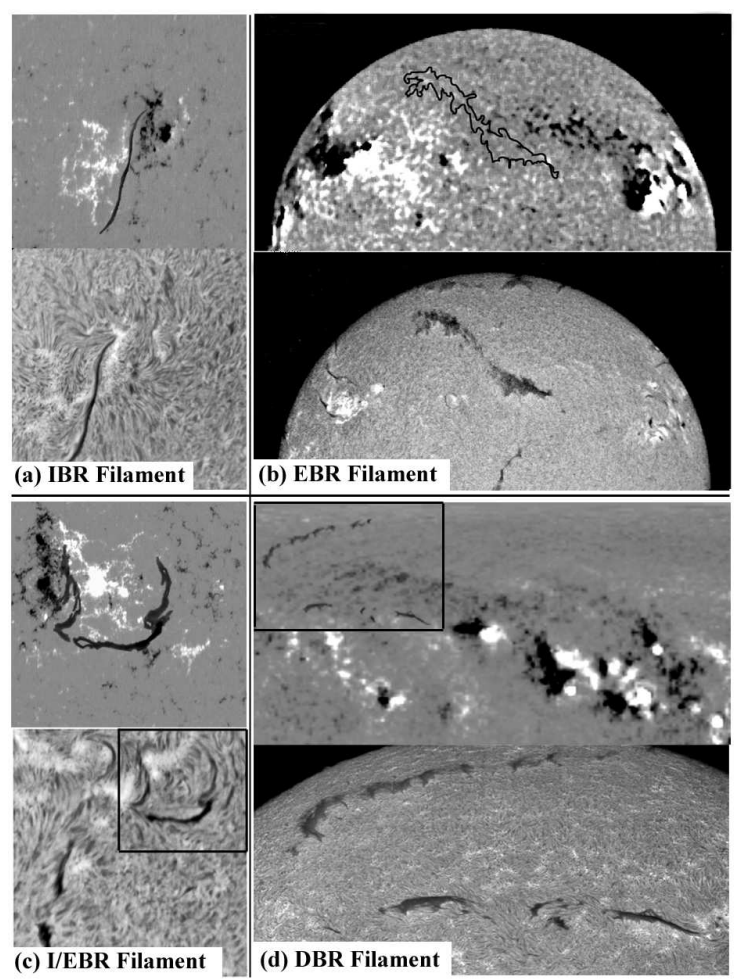

Fig. 3 Examples of the four categories of filaments defined by Mackay et al. (2008). In each of the panels (a)-(d), the bottom plot is an $\mathrm{H} \alpha$ image from the ORSO, while the top image shows the radial magnetic field derived from either (a)-(c) a full-disk magnetogram or (d) a synoptic magnetogram from Kitt Peak. Outlines of the $\mathrm{H} \alpha$ filaments are superimposed on each of the magnetograms. The dates of the observations are (a) $26^{\text {th }}$ June 1979, (b) $6^{\text {th }}$ May 1979, (c) $27^{\text {th }}$ September 1979 and (d) $14^{\text {th }}$ July 1979 . For panels (c) and (d) the areas enclosed by the boxes denote the corresponding area of (c) the magnetogram and (d) the $\mathrm{H} \alpha$ image. In panel (d) (top image) the low latitude activity complexes which will extend poleward over time and interact to produce diffuse regions of flux at high latitudes can be clearly seen. For a schematic representation of this figure see Chapter 2.

DBR and 13\% I/EBR). Only 7\% formed within a single bipole. These results show that large-scale filaments, namely those of the IF and QF type, preferentially form at sites of multiple bipole interactions. Very few of them occur within a single bipole. In addition by considering four distinct phases of the solar cycle, Mackay et al. (2008) showed that only EBR filaments exhibit any form of solar cycle dependence, with the other three types remaining essentially constant (see Figure 3 of Mackay et al. (2008)). This dependence showed that the number of EBR filaments varied in phase with the solar cycle and matches the solar cycle variation found by Zou et al. (2014), with more filaments at cycle maximum than minimum. Such a variation indicates that the formation of EBR filaments must be strongly related to the amount of magnetic flux on the Sun. 


\section{The Hemispheric Pattern of Solar Filament Channels and Filaments}

While the basic properties of solar filaments have long been known, filament channels and filaments have been classified more recently in terms of their chirality (Martin et al., 1992, 1994). This chirality may take one of two forms: dextral or sinistral. Dextral/sinistral filament channels and filaments have an axial magnetic field that points to the right/left when the main axis of the filament channel or filament is viewed from the positive polarity side of the PIL (see Figure 4). In force-free field models (e.g. Aulanier and Démoulin (1998); Mackay et al. (1999); van Ballegooijen et al. (2000); Mackay and van Ballegooijen (2005)) this chirality is directly related to the dominant sign of magnetic helicity that is contained within the filament channel and filament. A dextral filament or filament channel will contain dominantly negative helicity, while a sinistral one has positive helicity. The transport of filament channels and filaments across the solar surface (Section 1) is therefore an indication of the large-scale transport of magnetic helicity across the Sun (Yeates et al., 2008b), a key feature in explaining many eruptive phenomena.

A number of different techniques may be applied to determine the chirality of both filament channels and filaments depending on the resolution of the observations and strength of the underlying magnetic field. For a filament channel that does not contain a filament, high resolution $\mathrm{H} \alpha$ images are sufficient to resolve individual chromospheric fibrils and determine the chirality as long as the channel lies in a strong field region. In contrast for a channel in a weak field region, where fibril patterns are less distinct, magnetograms may also be used to aid the determination of chirality, by using them to determine the polarity of the magnetic elements from which the fibrils extend from or go into.

For filaments the chirality may be determined through a variety of techniques: (i) indirectly from the filament channel, as the filament is believed to lie in the extended field of the channel, (ii) directly through magnetic field measurements (Leroy et al., 1983), or, (iii) indirectly from the relationship of filaments to their barbs. Filaments with barbs may be classified as being either right-bearing or left-bearing depending on the direction in which the barbs extend out of the main filament axis. Martin and collaborators found a one-to-one correspondence where filaments in dextral channels have right-bearing barbs, and those in sinistral channels have left-bearing barbs. To date no studies have compared the chirality of filaments determined both directly (though magnetic field measurements) or indirectly (fibrils or barbs). Due to the lack of high resolution $\mathrm{H} \alpha$ data and direct measurements of magnetic fields within prominences, filaments are mostly classified using the relationship to barbs (Pevtsov et al., 2003; Yeates et al., 2007). Recently, a new technique for the determination of filament chirality has been described by Sheeley et al. (2013). This technique uses the plume like tails of coronal cells visible in the Fe XII 193 A line at $1.2 \mathrm{MK}$ to deduce the direction of the axial field up to a height of $50 \mathrm{Mm}$, significantly higher than that of $\mathrm{H} \alpha$. This new technique presents a powerful method due to the wide spread availability of observations in the Fe XII $193 \AA$ line. 

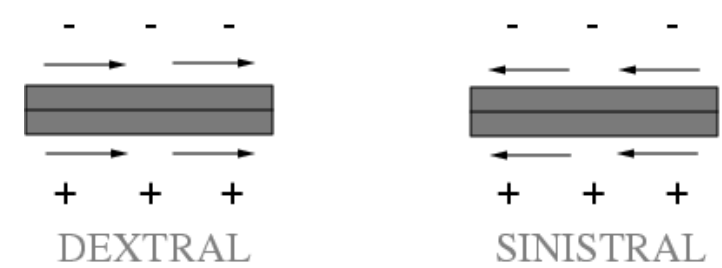

Fig. 4 The chirality of a filament channel is defined in terms of the direction of the magnetic field along the channel (denoted by arrows) when viewed by an observer on the positive polarity side of the channel. For a dextral (sinistral) channel, the magnetic field points to the right (left).

A surprising feature of the chirality of filament channels and filaments is that it displays an unusual large-scale hemispheric pattern: dextral/sinistral filaments dominate in the northern/southern hemispheres respectively (Martin et al., 1994; Zirker et al., 1997; Pevtsov et al., 2003; Yeates et al., 2007; Wang et al., 2013). This pattern is unusual as it is exactly opposite to that expected from differential rotation acting on a North-South coronal arcade. Although dextral/sinistral filaments dominate in the northern/southern hemisphere, observations show that exceptions to this pattern do occur. Any model which tries to explain the formation of filaments and filament channels must explain not only the origin of this hemispheric pattern but also why exceptions arise. Bernasconi et al. (2005) developed an automated detection software for filament barbs and the chirality of filaments. From applying this technique Martens et al. (2014) verified the existence of the hemispheric pattern, with the occurrence of exceptions for Cycle 23. However during the extended minimum between Cycles 23 and 24 the hemispheric pattern was sometimes present, but at other times absent. The reason for this near disappearance of the hemispheric pattern is unclear, but is probably due to the lack of emerging magnetic flux injecting helicity into the corona.

It is clear from the above discussion that solar filaments form or are found in a wide range of magnetic environments on the Sun, ranging from the rapidly evolving activity complexes to the slowly evolving streams of flux that extend out of the active latitudes towards the poles. To explain the formation of these filaments, observational studies and a wide range of theoretical models have been produced. The next section will consider observational case studies of the formation of filaments (Section 4). After discussing these, models of filaments formation will be discussed in Section 5. The models discussed in Section 5 will consider only global long-term magnetic field models for the formation of filament channels and filaments. For models that discuss the detailed thermodynamic processes and origin of the dense prominence plasma, see Chapters 7 and 10. The observations will then be used to clarify which models of filament formation are applicable to which filament formation locations (Section 7). 


\section{Observations of Filament Channel and Filament Formation}

To understand the magnetic environment and interactions leading to the formation of filament channels and filaments, it is useful to discuss test cases. To date, very few examples of filament channel formation have ever been observed, so the exact formation mechanism remains debatable. Within the published literature some papers show the formation of filament channels through surface effects that reconfigure pre-existing coronal fields, while in other papers flux emergence of horizontal flux ropes is deduced by the authors to play a critical role. Thus from interpreting the observations there appear to be two opposing views on how filament channels and filaments form. In this discussion we will consider the key observational features from case studies and determine whether the two views may be reconciled.

\subsection{Evidence of the Reconfiguration of Pre-Existing Coronal Fields in the Formation of Filament Channels}

Observations reported by Gaizauskas et al. (1997) and Gaizauskas et al. (2001) show that surface motions acting on pre-existing coronal fields play a critical role in the formation of filament channels and filaments. In the first case, an Intermediate Filament (IF) forms over a short period of a few days, while in the second a Quiescent Filament $(\mathrm{QF})$ forms over a period of months. In both cases the filaments form on PILs external to any single bipole and in the classification scheme of Section 2 would be classed as External Bipolar Regions Filaments. Although the two cases occur over very different time and length scales there are a number of important similarities.

Both cases begin with the emergence of a significant amount of magnetic flux in the form of an activity complex. Importantly however, no filaments form during the process of flux emergence. In fact, for the large scale QF the filament forms approximately 27 days after major flux emergence subsides. In both cases a necessary condition for the formation of the filament channels was flux convergence and cancellation at a PIL between separate bipolar regions. Such convergence and cancellation of flux was also shown to be important for filament formation in the papers by Martin (1998) and Gaizauskas (2002). Finally and most importantly, in each case a significant amount of magnetic shear was seen to build up in the activity complexes as they emerged. The redistribution of this non-potential field through surface motions towards the PIL produces a preferred direction of the coronal field above the PIL and plays a critical role in the formation of the filament channels (Mackay and Gaizauskas, 2003).

Figure 5 illustrates the main stages in the formation of a filament channel and IF over a period of five days between the $20-25^{\text {th }}$ July 1979 (see Figures 2 and 4 in Gaizauskas et al. (1997)). The formation of this southern-hemisphere IF involves the interaction of two distinct magnetic flux distributions, an old remnant region 

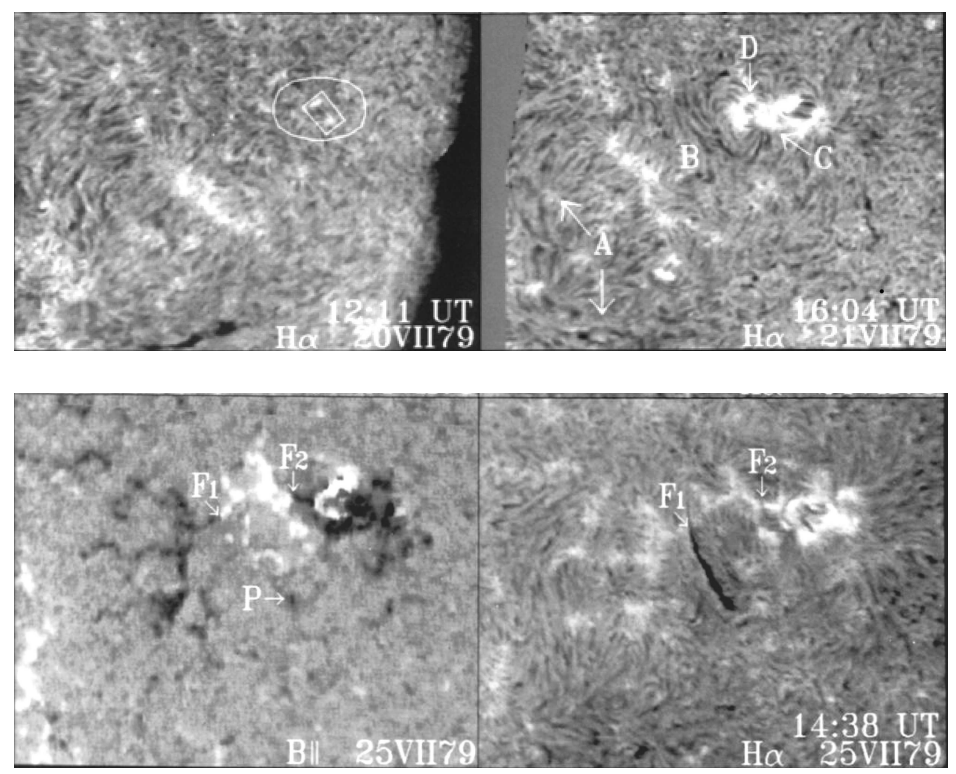

Fig. $5 \mathrm{H} \alpha$ and magnetogram images from Gaizauskas et al. (1997) of the formation of an Intermediate Filament between an old remnant region and a new emerging activity complex (inside oval). The $\mathrm{H} \alpha$ images correspond to $20^{\text {th }}$ (top left), $21^{\text {st }}$ (top right) and $25^{\text {th }}$ July 1979 where the filament channel formed on the $21^{\text {st }}$ but the filament (F1) did not form until the $25^{\text {th }}$ July 1979. In the magnetogram image on the $25^{t h}$, white represents positive and black negative flux.

( $\mathrm{M}^{c}$ Math 16159) and a new emerging region ( $\mathrm{M}^{c}$ Math 16166). In the $\mathrm{H} \alpha$ image of Figure 5 (top left) the bright North-South plage outlines the old remnant region; new magnetic flux emerges inside the oval between the $20^{\text {th }}$ and $21^{\text {st }}$ July. A key feature of this image is that the chromosphere is free of any strong patterns of magnetic fields surrounding the oval. Magnetic field observations show that the new emerging region is an activity complex (Gaizauskas et al., 1983; Benevolenskaya, 2005) made up of two or more sunspot pairs. Significantly, no filament forms near or around the activity complex during this period of rapid flux emergence. The key development in the formation of the filament channel occurs over a three hours period on the $21^{\text {st }}$ July (at the location denoted by B in upper right panel). Over this period a band of co-aligned fibrils form at the tail end of the new activity complex, between it and the old remnant region. These co-aligned fibrils indicate a magnetic field at this location with a dominant horizontal component, i.e. that a filament channel has formed. According to a model by Mackay et al. (1997) this pattern of co-aligned fibrils can only be explained by the extended non-potential magnetic field of the activity complex in which the field contains a large amount of positive helicity (correct sign for the southern hemisphere).

No filament forms as magnetic flux continues to emerge within the activity complex. The emergence ceases on the $23^{\text {rd }}$ July after which the trailing positive polarity of the activity complex disperses or diffuses out. This dispersion causes a conver- 
gence of flux between the old and new regions. In Figure 5 the distribution of magnetic flux (bottom left) and corresponding $\mathrm{H} \alpha$ image (bottom right) can be seen for the $25^{\text {th }}$ July. Five days after the complex started to emerge, cancellation of flux occurs at the point F1 (bottom right). After this cancellation the filament forms and passes through the location of flux cancellation. It survived for a full solar rotation and can clearly be seen to lie on a PIL which is external to any one bipolar region. Subsequent modeling by Mackay et al. (1997) showed that the resulting magnetic structure of the filament could only be explained by the interaction of the combined fields of both the old and new magnetic distributions. Both fields were highly non-potential, again with a significant amount of positive helicity which must have originated during the creation of the new activity complex. It is clear from the observations that this filament is of EBR type and reconfiguration of the previously emerged fields played a critical role in the formation of the filament. In the recent paper of Jeong et al. (2009) the authors present observations that support the idea that the non-potential fields and helicity of IF's originate during the emergence of activity complexes.

Gaizauskas et al. (2001) described a similar process of filament channel and filament formation between two neighboring activity complexes, but this time for a QF which is nearly $1 R_{\odot}$ in length. As with the previous case, the formation of the filament channel is attributed to the extended non-potential fields of the activity complexes. The filament only forms after major flux emergence ceases and the activity complexes converge and partially cancel with one another. In contrast to the IF case, which took 5 days to form, the large-scale case of the QF takes nearly one full solar rotation (27 days) to appear.

In both cases described above no stable filaments form during the periods of the highest rates of flux emergence, and the authors concluded that surface motions acting on pre-existing coronal fields play a critical role in the formation of stable filaments through the interaction of multiple bipoles. This result is consistent with the classification of filaments given in Section 2 where the majority of filaments are found to lie in magnetic configurations that involve more than one bipole. A key role of these surface motions is to redistribute the helicity which is seen to emerge in the early stages to form the filament channel (Gaizauskas et al., 1997, 2001; Mackay and Gaizauskas, 2003; Mackay and van Ballegooijen, 2005, 2006). In other observations, Gaizauskas (2002) show that convergence and cancellation of flux are important for filament channel formation (Martin, 1998). The study shows that, early on in the solar cycle, a unipolar region of flux has to extend $180^{\circ}$ around the Sun to interact and cancel with an opposite polarity region before a filament can form on that PIL. For this case the redistribution of flux, after emergence, is inferred to be a key process in the formation of the filament channel (Gaizauskas, 2008).

Observations by Wang and Muglach (2007) have supported the work of Gaizauskas et al. (1997, 2001). Wang and Muglach (2007) describe the formation of three filament channels and filaments (2 Intermediate or External Bipolar Regions Filaments and 1 Active Regions or I/EBR filament). The authors describe how fibrils which are initially normal to the PIL rotate to lie parallel to the PIL over a period of 1 to 2 days and in doing so form a filament channel. Through studying the evolution 
of the magnetic fields the authors deduce that flux cancellation as a result of supergranular convection plays a key role in the formation of the filament channels. They argue that this cancellation process between opposite polarity elements removes the normal component of the field but leaves the component parallel to the PIL which builds up gradually to form the axial field of the filament channel. In contrast to Gaizauskas et al. $(1997,2001)$ they do not observe any significant helicity resulting from the emergence of the active regions. On comparing the results of Gaizauskas et al. (1997) and Wang and Muglach (2007), while there are many similarities, there are also some differences in time scale. The clearest is the time difference required to form the filament channel. For Gaizauskas et al. (1997) the formation of the filament channel occurs over a 3 hour period and is attributed to the extended non-potential field of the activity complex containing a large amount of helicity. Cancellation of flux could not produce such a strongly sheared field over such a short period of time. In contrast, Wang and Muglach (2007) do not report any strong patterns of fibrils associated with helicity emerging in the active regions but rather form the filament channel over a period of 1 to 2 days in a much slower process of cancellation. Therefore there appear to be two complementary methods of forming a filament channel over different time scales.

Schmieder et al. (2004) studied the formation of a filament in the complex center of a decaying active region formed out of smaller individual components. They followed the evolution of three individual filament segments denoted F1, F2 and F3 over several days, and found that F1 and F2 gently merged into a single structure, as observed by a gradual filling in $\mathrm{H} \alpha$ of the gap between them. This merging was associated with mild EUV brightenings and with small $\mathrm{H} \alpha$ Doppler shifts at the merging point. While EUV brightenings are a good indicator of magnetic reconnection (see also Wang et al. (2013)), the flows revealed that the merging first took place by dynamic exchanges between the two progenitors, until they formed a single long stable filament. Two days later segments F2 and F3 came into contact and produced a confined flare, as evidenced by EUV post-flare loops (Deng et al., 2002). To determine the directions of the axial fields in the three filament segments, Schmieder et al. (2004) used the chirality rules for chromospheric fibrils and magnetic field polarity, the skew of the overlying coronal arcades, and the sense of twist in neighboring sunspots. It was then confirmed that when two filaments interact, magnetic reconnection takes place and leads to a merging when their chiralities are of the same sign, but leads to a flare when the chiralities are opposite. MHD simulations of such a process have been carried out by DeVore et al. (2005). It was also inferred that magnetic helicity must slowly accumulate prior to filament merging, as seen by the rotation of a small twisted sunspot close to the merging point. 


\subsection{Evidence of Emerging Horizontal Flux Tubes in Filament Formation}

It is clear from the above observations that surface effects play a critical role in forming the studied IFs and QFs (which are long stable structures). Lites and Low (1997) describe a different process for forming short, unstable active-region filaments. In Lites and Low (1997) the emergence of a $\delta$-spot is traced through vector magnetic field measurements using Advanced Stokes Polarimetry. Magnetic field vectors along part of the PIL within the emerging $\delta$-spot show a concave up or dipped magnetic structure (see Figure 1 of Lites and Low (1997); also see Lites (2005)). A small active region filament forms at this location. The filament was however unstable with a lifetime of only 2 days. Lites and Low (1997) suggest photospheric material is dragged up into the corona through the levitation process, as a horizontal flux rope emerges (Rust and Kumar (1994)).

A more recent example of the effect of evolving magnetic fields on the structure and stability of an active region filament is described by Okamoto et al. (2008) and Okamoto et al. (2009). In two papers, the authors present observations of a time series of vector magnetic fields taken by SOT underneath a pre-existing filament. The vector magnetic field measurements show a PIL with dominant horizontal field along it. This horizontal field probably represents that of the filament channel of the pre-existing filament. Over a period of 1.5 days the horizontal field vector changes from normal to inverse polarity and a dominant blue shift is observed. During this period the filament alters its appearance from a single structure, to a fragmented one and back again. Before returning to a single structure, brightenings are observed along the filament fragments in the $\mathrm{Ca}$ II $\mathrm{H}$ line.

From the observations the authors deduce two possible scenarios. In the first scenario they interpret the observations in terms of an emerging horizontal flux rope which fully emerges into the corona and occupies the position of the pre-existing filament. With this scenario the mass of the prominence originates from below the photosphere. In line with this scenario Lites et al. (2010) consider the formation of a filament channel within an active region and deduce that the formation is also due to the emergence of a flux rope. The second scenario interprets the brightenings in $\mathrm{Ca}$ II $\mathrm{H}$ as evidence for reconnection between the pre-existing filament and a new flux rope that emerges free of mass. The reconnection then produces a single structure along the PIL. A difficulty with both scenarios is that no simulations of magnetic flux emergence have been able to emerge a horizontal flux rope through the photosphere.

In contrast to that put forward by the authors, a third possibility also exists. As the top part of a flux rope emerges, a likely outcome is the emergence of sheared arcades. A coronal flux rope may then form out of these arcades through the process of reconnection. This reconnection may lift cool material into the corona. If the axial component of the emerging arcade lies in the same direction as that of the pre-existing filament channel, the new and old flux systems may join to produce a single structure. To consider which, if any of these three scenarios are correct, high 
resolution magnetic field observations at multiple levels in the solar atmosphere (e.g. photosphere, chromosphere and corona) are required. In the paper of Kuckein et al. (2012) the authors present a highly detailed study of vector magnetic fields at two heights around a compact active region filament. From the vector magnetic fields measurements they deduce that the magnetic configuration of the filament is that of a magnetic flux rope. While they show strong evidence for this, they cannot determine which scenario discussed above leads to the formation of the flux rope as the filament formed before the start of the vector magnetic field measurements. They do however find similarities in the evolution of the magnetic field to that described by Okamoto et al. (2008) and Okamoto et al. (2009).

\subsection{Summary of Observations}

The observations described in Section 4.1 and 4.2 provide evidence for filament formation arising from surface motions that reconfigure already existing coronal fields or, emerging flux tubes. So can the two methods be reconciled? The important distinction between these cases is the type and location of filaments formed in each case. For the cases in Section 4.1 surface motions play an important role in forming long stable Quiescent or Intermediate filaments which are External Bipolar Region Filaments, the dominant type of large-scale filament found at all latitudes on the Sun. In contrast, flux tubes emerging in a $\delta$-spot form an Active Region or Internal Bipolar Region Filaments which are unstable, lasting merely two days.

While it is difficult to draw general conclusions from a few specific observations, they indicate that two different mechanisms might form filaments in different magnetic environments on the Sun. Thus large stable filaments of the IF and QF type (External or Diffuse Bipolar Region) may require surface motions to gradually reconfigure pre-existing coronal fields, while small, short-lived ARFs (Internal Bipolar Region) may form due to flux emergence. To determine whether different mechanisms do produce different types of filaments at different locations on the Sun, the formation of filaments over a wide range of latitudes needs to be considered in detail. Observational programs required to do this will be briefly discussed in Section 7.

\section{Theoretical Models of Filament Formation}

Over the years many models have been constructed to describe the formation of filaments. These models vary from descriptive papers to full numerical MHD simulations and consider two main problems. First, how to obtain the correct dipped magnetic field configuration with dominant axial magnetic field that follows the hemispheric pattern, and secondly, the origin of the dense plasma. While the second question relates more to thermodynamics (Karpen et al. (2001), see Chapter 10), 
Table 1 Surface models of filament formation where the numbers attached to each paper correspond to the mechanisms given in Table 3

\begin{tabular}{ll}
\hline \hline Single Bipole & Multiple Bipoles \\
\hline van Ballegooijen and Martens (1989) $)^{1,3,4,10}$ & Kuperus $(1996)^{1,3,4}$ \\
DeVore and Antiochos $(2000)^{1,4}$ & Kuijpers $(1997)^{3,4,8,10}$ \\
& Mackay et al. $(1998)^{3,4,6,8,10}$ \\
& Galsgaard and Longbottom $(1999)^{3,4}$ \\
& van Ballegooijen et al. $(2000)^{1,4,10}$ \\
& Martens and Zwaan $(2001)^{3,4,10}$ \\
& Lionello et al. $(2002)^{8,10}$ \\
& DeVore et al. $(2005)^{1,3,4}$ \\
& Mackay and van Ballegooijen $(2005)^{1,4,8,10}$ \\
& Welsch et al. $(2005)^{3,4,8,10}$ \\
& Litvinenko and Wheatland $(2005)^{3,4,8,10}$ \\
& Yeates et al. $(2008 \mathrm{a})^{1,4,8,10}$ \\
& Xia et al. $(2014)^{3,4,8,10}$ \\
\hline
\end{tabular}

Table 2 Sub-surface models of filament formation where the numbers attached to each paper correspond to the mechanisms given in Table 3

\begin{tabular}{ll}
\hline \hline Single Bipole & Multiple Bipoles \\
\hline Low (1994) & van Ballegooijen and Martens $(1990)^{2,3,4,7}$ \\
Rust and Kumar (1994) $)^{7,9}$ & Priest et al. $(1996)^{2,3,4,6}$ \\
Gibson et al. $(2004)^{7,9}$ & Oliver $(1999)^{2,3,4,6}$ \\
Low and Hundhausen $(1995)^{7,9}$ & \\
Fan and Gibson $(2004)^{7,9}$ & \\
Fan and Gibson $(2006)^{7,9}$ & \\
Gibson and Fan $(2006)^{7,9}$ & \\
Magara (2006) $)^{7,9}$ & \\
Fan $(2009) 6,9$ & \\
\hline
\end{tabular}

this chapter is relevant to the first group of models. It is widely accepted that magnetic flux ropes are a suitable configuration to represent solar filaments; the main area of debate is how exactly these flux ropes may form. The various models which consider this may be broadly split into two distinct sub-groups: those employing surface effects to reconfigure coronal fields (Table 1) and those employing subsurface effects (Table 2). This split naturally arises from the discussion of the observations in Section 4. In these tables the surface/subsurface models have also been subdivided into those acting in single or multiple bipolar configurations in account of the observations discussed in Section 2. The list should only be regarded as representative and not exhaustive. Due to this, readers are recommended to search for other such papers in the literature. For each of the entries in Table 1 and 2 the numbers attached correspond to the various mechanisms that the models employ, as listed in Table 3. From the numbers attached to each model in Table 1 it is clear that surface models rely on a variety of mechanisms combined together. These include: differential rotation; shear flows along a PIL (differential rotation is just a weak shear 
Table 3 Mechanisms of Filament Formation

\begin{tabular}{ll}
\hline \multicolumn{1}{c}{ Surface Mechanisms } & \multicolumn{1}{c}{ Subsurface Mechanisms } \\
\hline (1) Differential Rotation (shear flows) & (2) Subsurface Motions \\
(3) Converging Flows & \\
(4) Magnetic Reconnection (atmosphere) & (5) Magnetic Reconnection (subsurface) \\
(6) Flux Emergence (bipoles) & (7) Flux Emergence (U-loops) \\
(8) Magnetic Helicity & (9) Magnetic Helicity \\
(10) Flux Cancellation/Diffusion & \\
\hline
\end{tabular}

flow); and converging flows onto a PIL. For some surface models diffusion of flux towards a PIL with subsequent cancellation plays the role of the converging flow. These mechanisms generally have to occur in a specific order to produce an axial magnetic field direction consistent with observations. In contrast some subsurface models apply a subsurface shear flow. In both surface and subsurface models magnetic reconnection is generally required to reconfigure the fields; the reconnection may occur either above or below the surface.

A common feature to both the surface and sub-surface models is flux emergence, but it is used in very different ways. For surface models, magnetic bipoles which emerge either untwisted or twisted are advected across the solar surface and reconfigured with other pre-existing coronal fields as discussed in the observations of Section 4.1. A key element in recent papers describing filament formation is that these bipoles are non-potential and include an initial magnetic helicity (Mackay and van Ballegooijen, 2005; Yeates et al., 2008a). In contrast, flux emergence for subsurface models is presumed to occur in the form of twisted U-loops (Section 4.2).

Whilst it is impractical to describe each of the models listed in Tables 1 and 2 in detail, key elements may be considered from a few selected cases. The cases chosen are picked solely for illustrative purposes. The key feature of any sub-surface model is described in the papers by Low (1994) and Rust and Kumar (1994). For these models a filament is formed when a horizontal twisted magnetic flux tube in the convective zone emerges due to magnetic buoyancy through the photosphere into the corona, dragging cool dense material with it (Gibson and Fan, 2006). In flux emergence simulations where the authors use only buoyancy and magnetic buoyancy instabilities, it is found that the axis of the flux rope does not rise through the photosphere (Archontis et al., 2004; Archontis, 2008; Murray et al., 2006; Galsgaard et al., 2007). Although the axis and U-loops of the emerging tube do not rise to coronal heights, the process of flux emergence may still produce a coronal flux rope with dips. A flux rope may form through the reconnection of emerged sheared field lines that lie above the emerging tubes axis (Manchester et al., 2004; Magara, 2006; Archontis and Török, 2008; Fan, 2009).

In contrast, one of the first surface models, by van Ballegooijen and Martens (1989), considers shearing motions acting on a coronal arcade in a bipolar configuration. The footpoints of the arcade are sheared in such a way that their separation increases and an axial field component is produced along the PIL (see Figures 6a and $6 \mathrm{~b}$ ). In principle this shear could be a result of solar differential rotation or by other 


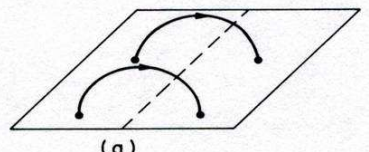

(a)

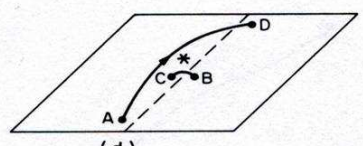

(d)

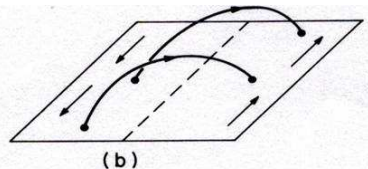

(b)

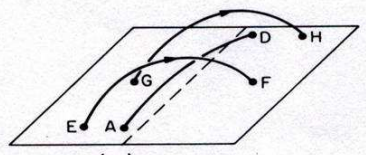

(e)

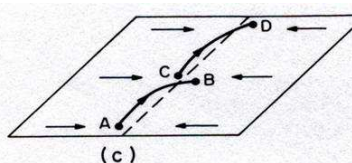

(c)

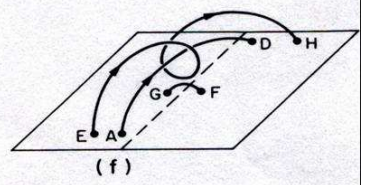

Fig. 6 Example of the formation of a filament's axial magnetic field through shearing motions, convergence, and reconnection as put forward by van Ballegooijen and Martens (1989).

shear flows on the Sun. Next convergence, or diffusion of the flux towards the PIL, brings the foot points together where they may reconnect to produce, a long axial field line along the PIL and also a small loop which submerges through the surface (Figures $6 \mathrm{c}$ and $6 \mathrm{~d}$ ). Subsequent repetition of this process creates dipped magnetic field lines consistent with the topology required for filaments (Figures 6e and 6f). This idea was developed further by Martens and Zwaan (2001) who put forward a "head-to-tail" linkage model for the formation of filaments through the interaction of multiple bipoles. While Martens and Zwaan considered this in a conceptual model, Mackay et al. (2000) and Mackay and van Ballegooijen (2001) carried out numerical simulations of a similar process.

An alternative method of forming a strongly sheared magnetic structure was proposed by DeVore and Antiochos (2000) using a single bipolar configuration (also see Antiochos et al. (1994)). In this model, a bipolar magnetic field distribution is subjected to a strong shearing motion parallel to the PIL, however, no converging flow is applied. Once the footpoints of the field lines are sheared a distance comparable to the bipole width, an untwisted dipped magnetic configuration forms. The authors show that through further shearing of the dipped field lines the initially untwisted field may form a helical structure similar to that of van Ballegooijen and Martens (1989) through a two stage reconnection process. Therefore, in contrast to van Ballegooijen and Martens (1989) and Martens and Zwaan (2001), DeVore and Antiochos (2000) do not rely on convergence and cancellation of flux to produce the helical field.

From the discussion above it is clear that a wide range of theoretical models exist to explain the 3D magnetic structure of solar filaments. At the present time none of these models may be ruled out. However, by combining the observations discussed in Sections 4.1 and 4.2 it may be argued that some models are more relevant than others for the formation of large stable filaments (Quiescent and Intermediate) compared to Active Region filaments. A full discussion along with the presented hypothesis will be carried out in Section 7 . 


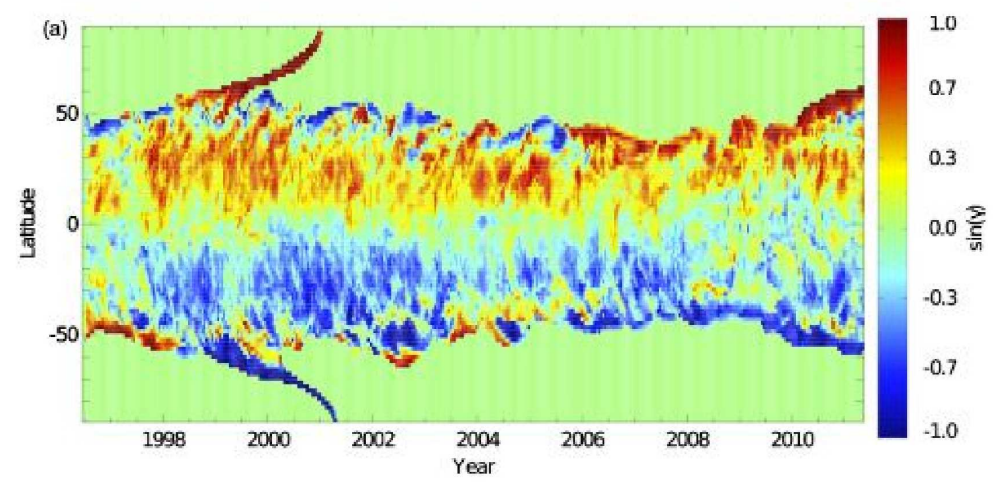

Fig. 7 Butterfly diagram over Cycle 23 showing the longitude-averaged skew $\sin \gamma$ measured at height $r=1.033 R_{\odot}$ (from Yeates \& Mackay (2012)). In the figure red represents dextral skew and blue sinistral skew.

\section{Origin of the Hemispheric Pattern of Filaments}

Any model which tries to explain the origin of a filament's magnetic field must also explain why this magnetic field exhibits a hemispheric pattern. Initial studies have considered the origin of the hemispheric pattern by modeling the evolution of either idealised magnetic distributions (Mackay and van Ballegooijen, 2001, 2005) or observed distributions that can be compared directly with subsequent measurements (van Ballegooijen et al., 1998; Mackay et al., 2000). To date the most detailed investigation into the origin of the chirality of filaments has been carried out by Yeates et al. (2007) and Yeates et al. (2008a). To carry out the comparison the authors first determined the location and chirality of 109 filaments over a 6 month period. They then developed a new technique to model the continuous long term, global evolution of the Sun's magnetic field from synoptic magnetogram observations. A key feature of the simulations was that they considered the long term helicity transport across the solar surface from low to high latitudes.

Applying this technique, Yeates et al. (2008a) carried out a direct one-to-one comparison of the chirality produced by the model with the observed chirality of the filaments at the exact location that each filament was observed. It was found that, if the transport effects of differential rotation, meridional flow, and surface diffusion are combined with newly emerging bipoles in the northern/southern hemisphere already containing negative/positive helicity, then a $96 \%$ agreement can be obtained between the observed chirality of the filaments and that produced by the model. The agreement was equally good for both the dominant and minority chirality in each hemisphere.

In a further study, Yeates \& Mackay (2012) simulated the global non-potential corona for the entire length of Cycle 23 where the coronal magnetic field is continuously evolved over a 15 year period and 1838 active regions containing helicity are 
emerged. Results from the simulation can be seen in Figure 7 where the latitudinal distribution of chirality in both hemispheres (red $\sim$ dextral, blue $\sim$ sinistral) can be seen. Below $\pm 50^{\circ}$ latitude the dominant pattern of chirality predominates, i.e., dextral in the northern hemisphere and sinistral in the southern hemisphere, although the overall pattern has significant fluctuations (minority chirality exists at all latitudes). During the rush-to-the-poles between 1998 and 2001 the polar crowns exhibit the dominant chirality pattern. In contrast during the period of low activity from 2007 to 2010, there is a more mixed chirality at lower latitudes. However from 2010 onward, the dominant chirality dominates at high latitudes once more, continuing into Cycle 24. Interestingly until 1998 and during the declining phase of cycle 23 (2001 to 2006), there is a tendency for minority chirality to occur on the high-latitude PILs (sinistral in the north, dextral in the south). The initial phase of minority chirality at high-latitudes in both hemispheres until 1998 is due the initial condition which is a potential field. Once this is removed by the transport of helicity poleward which occurs over a 2 year time period the correct chirality is found at high latitudes in the rising phase. In contrast in the declining phase the pattern is a true feature of the model and is not due to the initial condition. Thus far, no detailed observational studies of filament chirality have been carried out in the declining phase of the solar cycle to test these predictions.

In contrast to the simulations of Yeates \& Mackay (2012) where magnetic helicity is sporadically injected into the corona through flux emergence, Antiochos (2013) proposed a new helicity-condensation model for the formation of filament channels. In this model filament channels form through a multi-stage process of helicity injection, transfer, and condensation that acts on the chromospheric and coronal magnetic fields. First helicity is injected into the overlying atmosphere by small-scale, vortical motions associated with both granular and supergranular convection. Assuming that the motions are predominantly counterclockwise in the northern hemisphere and clockwise in the southern, then the resultant magnetic twist is dextral in the north and sinistral in the south. Next reconnection within magnetically unipolar regions transfers the twist field out to the extent of the unipolar region. At the boundaries between regions of opposite magnetic polarity, the twist then accumulates at the PIL and results is an increasingly strong axial field and filament channel.

The paper of Mackay et al. (2014) considered the large-scale consequences of the helicity-condensation process, in conjunction to other flux-transport processes that affect filament-channel formation. Using a large-scale, spatially averaged representation of the helicity condensation process, the simulations show that on a northsouth oriented PIL the mechanisms applied by both Yeates \& Mackay (2012) and Antiochos (2013) inject the same sign of helicity, which reproduces the dominant hemispheric pattern of filaments. In contrast on a high-latitude east-west oriented polar crown or sub-polar crown PIL, the model of Antiochos (2013) adds a new feature. If the vorticity of the cells is approximately $2-3$ times greater than the local differential-rotation gradient then the helicity condensation can overcome the incorrect sign of helicity injection from differential rotation. Finally in the declining phase of the cycle, as a bipole interacts with the polar field, in some cases helic- 
ity condensation can reverse the effect of differential rotation along the east-west lead arm that surround bipoles, but not in all cases. The results show that the magnetic helicity injection and condensation model of Antiochos (2013), in conjunction with the mechanisms used in Yeates et al. (2008a), is a viable explanation for the hemispheric pattern of filaments. In principle, if future observational studies of filament chirality in the declining phase disagree with the simulation results of Yeates \& Mackay (2012) then the helicity condensation model may resolve any potential incompatibilities.

\section{Discussion and Future Observations}

So far we have discussed a number of properties of solar filaments and filament channels. These properties have ranged from observations and locations of their formation, to the wide variety of theoretical models used to explain them and their hemispheric pattern. We now turn our attention to tying all of these observations together, by forming a unifying hypothesis, to quantify where and at what locations the mechanisms and models discussed in Section 5 are appropriate. The aim of this hypothesis is to stimulate new observational studies to test it.

From the observations of large-scale stable filaments discussed in Section 2 and 4 it can be seen that IF and QF preferentially, but not exclusively, form in magnetic configurations involving multiple bipole interactions (92\%). While none of the models listed in Table 1 or 2 can be ruled out, it is clear that those involving multiple bipole interactions are the most appropriate for these types of filament.

The question now turns to whether the IF and QF are formed due to surface motions acting on pre-existing coronal fields or whether they are due to sub-surface processes. The observations of Gaizauskas et al. (1997), Gaizauskas et al. (2001) and Wang and Muglach (2007) show examples of IF and QF that do not form during the emergence of flux but rather after convergence and cancellation of individual bipoles. Therefore, it may be argued that convergence leading to subsequent cancellation and reconnection (i.e., items 3, 4 and 10 in Table 3) are the mechanisms that result in the formation of large stable filaments found on the Sun. For these filaments flux rope emergence does not appear to play a major role, however the injection of magnetic helicity due to the process of flux emergence at an earlier time may play a key role in producing the filament channels. For those large stable filaments that do form within single bipoles (7\%) shear flows such as applied by DeVore and Antiochos (2000) may play a key role in the formation. The models in Table 1 which include these mechanisms appear to be the most appropriate to explain IF and QF. At the present time no further distinction can be made between these models.

In contrast the observations by Lites and Low (1997), Okamoto et al. (2009) and Lites et al. (2010) suggest that small-scale unstable active region filaments may be formed as the result of flux rope emergence dragging cool dense photospheric plasma into the corona. While this is a possibility, most numerical simulations of emerging flux ropes fail to lift the axis and cool material of the original flux tube 
into the corona. Therefore it remains unclear whether such a process may occur. In contrast, many authors have shown that during the process of flux emergence, after the top of the flux rope has emerged, magnetic reconnection or helicity injection (Manchester et al., 2004; Magara, 2006; Archontis and Török, 2008; Fan, 2009) may reconfigure the emerged coronal arcade to produce a secondary coronal flux rope. During the formation of the secondary flux rope the reconnection may then lift cool dense material to coronal heights. Therefore while emerging flux appears to be important for the formation of active region filaments a key element may still be atmospheric reconnection of pre-emerged fields. To resolve this issue new observational studies similar to that of Kuckein et al. (2012), but which follow the early stages of the formation of the AR filament are required.

From the discussion above it appears that different formation mechanisms may apply to different types of filaments. Quiescent filaments and Intermediate filaments which mainly fall into the Exterior and Diffuse bipolar region types rely on surface effects acting on coronal fields. In contrast, for active region filaments a strong possibility is the emergence of flux ropes or the formation of flux ropes during emergence as a result of coronal reconnection. Therefore it is useful to distinguish between IF and $\mathrm{QF}$, compared to ARF as they may have a different formation mechanism.

The formation, structure, and evolution of solar filaments is an important part of our understanding of coronal physics and the behavior of magnetic fields as they are transported across the solar surface. Present evidence suggests that a number of different mechanisms may be taking place in magnetic different environments. A better understanding of the formation of prominences requires multi-wavelength observations of prominences situated over a wide range of latitudes, from the active region belts up to the polar crowns. It is imperative to determine whether different formation mechanisms occur at different latitudes on the Sun. To distinguish this, spectral lines from $H \alpha$ to X-rays along with magnetic information are needed to provide full coverage of the wavelength ranges associated with the formation and structure of filaments. A key aspect of this study is being able to determine where and when a long-lived filament might form. Therefore, maintenance of existing synoptic data sets is a vital part of advanced studies of prominence formation. In addition, new observational studies are required to understand the magnitude and distribution of vortical motions occurring in convective cells, as these motions may be a missing piece in our understanding of magnetic helicity generation and transport across the Sun.

Acknowledgements DHM would like to thank the members of the two ISSI teams on solar prominences lead by Nicolas Labrosse for their stimulating discussions. In particular DHM would like to give special thanks to Aad van Ballejooijen, Judy Karpen, Jose Luis Ballester, Brigitte Schmieder and Guillaume Aulanier who have all helped develop my understanding of solar prominences and aided my ability to write the present chapter. DHM would also like to thank STFC and the Leverhulme Trust for their financial support. 


\section{References}

Antiochos, S. K.: (2013), Helicity Condensation as the Origin of Coronal and Solar Wind Structure, ApJ , 772, 72

Antiochos, S. K., Dahlburg, R. B., \& Klimchuk, J. A.: (1994), The magnetic field of solar prominences, ApJ , 420, L41

Archontis, V.: (2008), Magnetic flux emergence in the Sun, Journal of Geophysical Research (Space Physics), 113, 3

Archontis, V., Török, T.: (2008), Eruption of magnetic flux ropes during flux emergence, A\&A , 492, L35

Archontis, V., Moreno-Insertis, F., Galsgaard, K., Hood, A., \& O’Shea, E.: (2004), Emergence of magnetic flux from the convection zone into the corona, A\&A, 426, 1047

Aulanier, G., \& Demoulin, P.: (1998), 3-D magnetic configurations supporting prominences. I. The natural presence of lateral feet, A\&A, 329, 1125

Babcock, H. W., \& Babcock, H. D.: (1955), The Sun's Magnetic Field, 1952-1954. ApJ , 121, 349

Benevolenskaya, E. E. : (2005), The Formation and Evolution of Complexes of Activity, Activity Nests and the Large-scale Connectivity in the Solar Corona, in Large-scale Structures and their Role in Solar Activity, 346, 129

Bernasconi, P. N., Rust, D. M., \& Hakim, D.: (2005), Advanced Automated Solar Filament Detection And Characterization Code: Description, Performance, And Results, Sol. Phys. , 228, 97

Coffey, H. E., \& Hanchett, C. D.: (1998), Digital "Cartes Synoptiques de la Chromosphere Solaire et Catalogues des Filaments et des Centres d'Activite”, IAU Colloq. 167: New Perspectives on Solar Prominences, 150, 488

d'Azambuja, L. and d'Azambuja, M., Ann. Obs. Paris-Meudon 6, 7 (1948)

Deng, Y., Lin, Y., Schmieder, B., \& Engvold, O.: (2002), Filament activation and magnetic reconnection,Sol. Phys. , 209, 153

DeVore, C. R., \& Antiochos, S. K.: (2000), Dynamical Formation and Stability of Helical Prominence Magnetic Fields , ApJ , 539, 954

DeVore, C. R., Antiochos, S. K., \& Aulanier, G.: (2005), Solar Prominence Interactions, ApJ , 629, 1122

Engvold, O.: (1998), Observations of Filament Structure and Dynamics (Review), IAU Colloq. 167: New Perspectives on Solar Prominences, 150, 23

Fan, Y.: (2009), The Emergence of a Twisted Flux Tube into the Solar Atmosphere: Sunspot Rotations and the Formation of a Coronal Flux Rope, ApJ , 697, 1529

Fan, Y., \& Gibson, S. E.: (2004), Numerical Simulations of Three-dimensional Coronal Magnetic Fields Resulting from the Emergence of Twisted Magnetic Flux Tubes ApJ , 609, 1123

Fan, Y., \& Gibson, S. E.: (2006), On the Nature of the X-Ray Bright Core in a Stable Filament Channel, ApJ , 641, L149

Foukal, P.: (1971), Morphological Relationships in the Chromospheric H $\alpha$ Fine Structure Sol. Phys. , 19, 59 


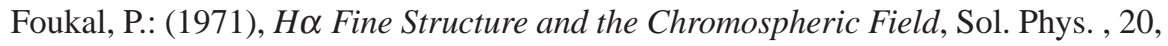
298

Gaizauskas, V.: (1998), Filament Channels: Essential Ingredients for Filament Formation (Review), IAU Colloq. 167: New Perspectives on Solar Prominences, 150, 257

Gaizauskas, V.: (2002), Formation of a Switchback During the Rising Phase of Solar Cycle 21, Sol. Phys. , 211, 179

Gaizauskas, V.: (2008), Development of Flux Imbalances in Solar Activity Nests and the Evolution of Filament Channels, ApJ , 686, 1432

Gaizauskas, V., Harvey, K. L., Harvey, J. W., \& Zwaan, C.: (1983), Large-scale patterns formed by solar active regions during the ascending phase of cycle 21 ApJ , 265, 1056

Gaizauskas, V., Mackay, D. H., \& Harvey, K. L.: (2001), Evolution of Solar Filament Channels Observed during a Major Poleward Surge of Photospheric Magnetic Flux , ApJ , 558, 888

Gaizauskas, V., Zirker, J. B., Sweetland, C., \& Kovacs, A.: (1997), Formation of a Solar Filament Channel ApJ , 479, 448

Galsgaard, K., \& Longbottom, A. W.: (1999), Formation of Solar Prominences by Flux Convergence, ApJ , 510, 444

Galsgaard, K., Archontis, V., Moreno-Insertis, F., \& Hood, A. W.: (2007), The Effect of the Relative Orientation between the Coronal Field and New Emerging Flux. I. Global Properties, ApJ , 666, 516

Gibson, S. E., \& Fan, Y.: (2006), Coronal prominence structure and dynamics: A magnetic flux rope interpretation, Journal of Geophysical Research (Space Physics), 111, 12103

Gibson, S. E., Fan, Y., Mandrini, C., Fisher, G., \& Demoulin, P.: (2004), Observational Consequences of a Magnetic Flux Rope Emerging into the Corona, ApJ , 617,600

Hyder, C. L.: (1965), The Polarization of Emission Lines in Astronomy. II. Prominence Emission-Line Polarization and Prominence Magnetic Fields. ApJ , 141, 1374

Jeong, H., Chae, J., \& Moon, Y.-J.: (2009), Magnetic Helicity Injection During the Formation of an Intermediate Filament, Journal of Korean Astronomical Society, 42,9

Karachik, N. V., \& Pevtsov, A. A.: (2014), Properties of Magnetic Neutral Line Gradients and Formation of Filaments, Sol. Phys. , 289, 821

Karpen, J. T., Antiochos, S. K., Hohensee, M., Klimchuk, J. A., \& MacNeice, P. J.: (2001), Are Magnetic Dips Necessary for Prominence Formation? ApJ , 553, L85

Kuckein, C., Martínez Pillet, V., \& Centeno, R.: (2012), An active region filament studied simultaneously in the chromosphere and photosphere. II. Doppler velocities, A\&A , 539, A131

Kuijpers, J.: (1997), A Solar Prominence Model ApJ , 489, L201

Kuperus, M.: (1996), The Double Inverse Polarity Paradigm-The Sign of Magnetic Fields in Quiescent Prominences, Sol. Phys. , 169, 349 
Kuperus, M., \& Raadu, M. A.: (1974), The Support of Prominences Formed in Neutral Sheets, A\&A , 31, 189

J.-L. Leroy, in Dynamics and Structure of Quiescent SolarProminences, ed. by E.R. Priest (Kluwer, Dordrecht, 1989), p. 77

Leroy, J. L., Bommier, V., \& Sahal-Brechot, S.: (1983), The magnetic field in the prominences of the polar crown Sol. Phys. , 83, 135

Li, K. J.: (2010), Latitude migration of solar filaments , MNRAS , 405, 1040

Lionello, R., Mikić, Z., Linker, J. A., \& Amari, T.: (2002),Magnetic Field Topology in Prominences , ApJ , 581, 718

Lites, B. W.: (2005),Magnetic Flux Ropes in the Solar Photosphere: The Vector Magnetic Field under Active Region Filaments ApJ , 622, 1275

Lites, B. W., \& Low, B. C.: (1997),Flux Emergence and Prominences: a New Scenario for 3-DIMENSIONAL Field Geometry Based on Observations with the Advanced Stokes Polarimeter, Sol. Phys. , 174, 91

Lites, B. W., Kubo, M., Berger, T., et al.: (2010), Emergence of Helical Flux and the Formation of an Active Region Filament Channel, ApJ , 718, 474

Litvinenko, Y. E.: (2000), On the Magnetic Field Orientation and Plasma Flows in Solar Filament Barbs , Sol. Phys. , 196, 369

Litvinenko, Y. E., \& Wheatland, M. S.: (2005), A Simple Dynamical Model for Filament Formation in the Solar Corona, ApJ , 630, 587

López Ariste, A., Aulanier, G., Schmieder, B., \& Sainz Dalda, A.: (2006), First observation of bald patches in a filament channel and at a barb endpoint, A\&A, 456, 725

Low, B. C.: (1994), Magnetohydrodynamic processes in the solar corona: Flares, coronal mass ejections, and magnetic helicity, Physics of Plasmas, 1, 1684

Low, B. C., \& Hundhausen, J. R.: (1995), Magnetostatic structures of the solar corona. 2: The magnetic topology of quiescent prominences, ApJ , 443, 818

Mackay, D. H., \& Gaizauskas, V.: (2003), Helicity as a Component of Filament Formation, Sol. Phys. , 216, 121

Mackay, D. H., \& van Ballegooijen, A. A.: (2001), A Possible Solar Cycle Dependence to the Hemispheric Pattern of Filament Magnetic Fields?, ApJ , 560, 445

Mackay, D. H., \& van Ballegooijen, A. A.: (2005), New Results in Modeling the Hemispheric Pattern of Solar Filaments , ApJ , 621, L77

Mackay, D. H., \& van Ballegooijen, A. A.: (2006), Models of the Large-Scale Corona. I. Formation, Evolution, and Liftoff of Magnetic Flux Ropes , ApJ , 641, 577

Mackay, D. H., Gaizauskas, V., Rickard, G. J., \& Priest, E. R.: (1997), Force-free and Potential Models of a Filament Channel in Which a Filament Forms, ApJ , 486, 534

Mackay, D. H., Priest, E. R., Gaizauskas, V., \& van Ballegooijen, A. A.: (1998), Role of Helicity in the Formation of Intermediate Filaments, Sol. Phys. , 180, 299

Mackay, D. H., Longbottom, A. W., \& Priest, E. R.: (1999), Dipped Magnetic Field Configurations Associated with Filaments and Barbs, Sol. Phys. , 185, 87 
Mackay, D. H., Gaizauskas, V., \& van Ballegooijen, A. A.: (2000), Comparison of Theory and Observations of the Chirality of Filaments within a Dispersing Activity Complex, ApJ , 544, 1122

Mackay, D. H., Gaizauskas, V., \& Yeates, A. R.: (2008), Where Do Solar Filaments Form?: Consequences for Theoretical Models, Sol. Phys. , 248, 51

Mackay, D. H., DeVore, C. R., \& Antiochos, S. K.: (2014), , apj, 784, 164

Magara, T.: (2006), Dynamic and Topological Features of Photospheric and Coronal Activities Produced by Flux Emergence in the Sun, ApJ , 653, 1499

Malherbe, J.-M.: (1989), The formation of solar prominences, Dynamics and Structure of Quiescent Solar Prominences, 150, 115

Manchester, W., IV, Gombosi, T., DeZeeuw, D., \& Fan, Y.: (2004), Eruption of a Buoyantly Emerging Magnetic Flux Rope , ApJ , 610, 588

Martens, P. C., \& Zwaan, C.: (2001), Origin and Evolution of Filament-Prominence Systems, ApJ , 558, 872

Martens, P. C., Yeates, A. R., \& Pillai, K. G.: (2014),, IAU Symposium, 300, 135

Martin, S. F.: (1998), Conditions for the Formation and Maintenance of Filaments (Invited Review) , Sol. Phys. , 182, 107

Martin, S. F., Marquette, W. H., \& Bilimoria, R. (1992), The Solar Cycle Pattern in the Direction of the Magnetic Field along the Long Axes of Polar Filaments" , The Solar Cycle, 27, 53

Martin, S. F., Bilimoria, R., \& Tracadas, P. W.: (1994), Magnetic field configurations basic to filament channels and filaments, Solar Surface Magnetism, 303

Martres, M. J., Michard, R., \& Soru-Iscovici: (1966), Étude morphologique de la structure magnétique des régions actives en relation avec les phénomènes chromosphériques et les éruptions solaires. II. Localisation des plages brillantes, filaments et éruptions, Annales d'Astrophysique, 29, 249

McIntosh, P. S.: (1972), Solar magnetic fields derived from hydrogen alpha filtergrams. , Reviews of Geophysics and Space Physics, 10, 837

Minarovjech, M., Rybansky, M., \& Rusin, V.: (1998), Prominences and the Green Corona Over the Solar Activity Cycle, Sol. Phys. , 177, 357

Mouradian, Z., \& Soru-Escaut, I.: (1994), A new analysis of the butterfly diagram for solar filaments., A\&A , 290, 279

Murray, M. J., Hood, A. W., Moreno-Insertis, F., Galsgaard, K., \& Archontis, V.: (2006), 3D simulations identifying the effects of varying the twist and field strength of an emerging flux tube, A\&A , 460, 909

Okamoto, T. J., Tsuneta, S., Lites, B. W., et al.: (2008), Emergence of a Helical Flux Rope under an Active Region Prominence, ApJ , 673, L215

Okamoto, T. J., Tsuneta, S., Lites, B. W., et al.: (2009), Prominence Formation Associated with an Emerging Helical Flux Rope, ApJ , 697, 913

Oliver, R., Čadež , V. M., Carbonell, M., \& Ballester, J. L.: (1999), Coronal potential magnetic fields from photospheric sources with finite width, A\&A , 351, 733

Pevtsov, A. A., Balasubramaniam, K. S., \& Rogers, J. W.: (2003), Chirality of Chromospheric Filaments, ApJ , 595, 500 
Pevtsov, A. A., Panasenco, O., \& Martin, S. F.: (2012), Coronal Mass Ejections from Magnetic Systems Encompassing Filament Channels Without Filaments, Sol. Phys. , 277, 185

Pintér, T., Rybanský, M., \& Dorotovič, I.: (2014), The polar belts of prominence occurence as an indicator of the solar magnetic field reversal, IAU Symposium, 300,456

Priest, E. R., van Ballegooijen, A. A., \& Mackay, D. H.: (1996), A Model for Dextral and Sinistral Prominences, ApJ , 460, 530

Rust, D. M.: (1967), Magnetic Fields in Quiescent Solar Prominences. I. Observations , ApJ , 150, 313

Rust, D. M., \& Kumar, A.: (1994), Helical magnetic fields in filaments, Sol. Phys. , 155,69

Schmieder, B., Mein, N., Deng, Y., et al.: (2004), Magnetic changes observed in the formation of two filaments in a complex active region: TRACE and MSDP observations", Sol. Phys. , 223, 119

Sheeley, N. R., Jr., Martin, S. F., Panasenco, O., \& Warren, H. P.: (2013), Using Coronal Cells to Infer the Magnetic Field Structure and Chirality of Filament Channels, ApJ , 772, 88

Shimojo, M., Yokoyama, T., Asai, A., Nakajima, H., \& Shibasaki, K.: (2006), One Solar-Cycle Observations of Prominence Activities Using the Nobeyama Radioheliograph 1992-2004, PASJ , 58, 85

Tang, F.: (1987), Quiescent prominences - Where are they formed? Sol. Phys. , 107, 233

Topka, K., Moore, R., Labonte, B. J., \& Howard, R.: (1982), Evidence for a poleward meridional flow on the sun, Sol. Phys. , 79, 231

van Ballegooijen, A. A.: (2004), Observations and Modeling of a Filament on the Sun, ApJ , 612, 519

van Ballegooijen, A. A., \& Martens, P. C. H.: (1989), Formation and eruption of solar prominences, ApJ , 343, 971

van Ballegooijen, A. A., \& Martens, P. C. H.: (1990), Magnetic fields in quiescent prominences , ApJ , 361, 283

van Ballegooijen, A. A., Cartledge, N. P., \& Priest, E. R.: (1998), Magnetic Flux Transport and the Formation of Filament Channels on the Sun, ApJ , 501, 866

van Ballegooijen, A. A., Priest, E. R., \& Mackay, D. H.: (2000), Mean Field Model for the Formation of Filament Channels on the Sun”, ApJ , 539, 983

Wang, Y.-M., \& Muglach, K.: (2007), On the Formation of Filament Channels, ApJ , 666, 1284

Wang, Y.-M., \& Muglach, K.: (2013), Transient Brightenings Associated with Flux Cancellation along a Filament Channel ApJ , 763, 97

Wang, Y.-M., Sheeley, N. R., Jr., \& Stenborg, G.: (2013), Fe XII Stalks and the Origin of the Axial Field in Filament Channels, ApJ , 770, 72

Welsch, B. T., DeVore, C. R., \& Antiochos, S. K.: (2005), Magnetic Reconnection Models of Prominence Formation, ApJ , 634, 1395 
Xia, C., Keppens, R., \& Guo, Y.: (2014), Three-dimensional Prominence-hosting Magnetic Configurations: Creating a Helical Magnetic Flux Rope, ApJ , 780, 130

Yeates, A. R., \& Mackay, D. H.: (2009), Modelling the Global Solar Corona: III. Origin of the Hemispheric Pattern of Filaments", Sol. Phys. , 254, 77

Yeates, A. R., \& Mackay, D. H.: (2012), Chirality of High-latitude Filaments over Solar Cycle 23, ApJ , 753, L34

Yeates, A. R., Mackay, D. H., \& van Ballegooijen, A. A.: (2007), Modelling the Global Solar Corona: Filament Chirality Observations and Surface Simulations, Sol. Phys. , 245, 87

Yeates, A. R., Mackay, D. H., \& van Ballegooijen, A. A.: (2008), Modelling the Global Solar Corona II: Coronal Evolution and Filament Chirality Comparison , Sol. Phys. , 247, 103

Yeates, A. R., Mackay, D. H., \& van Ballegooijen, A. A.: (2008), Evolution and Distribution of Current Helicity in Full-Sun Simulations, ApJ , 680, L165

Zirker, J. B., Martin, S. F., Harvey, K., \& Gaizauskas, V.: (1997), Global Magnetic Patterns of Chirality, Sol. Phys., 175, 27

Zou, P., Li, Q.-X., \& Wu, N.: (2014), Non-linear analysis of the long-term behaviour of solar filaments , MNRAS , 437, 38 